\title{
Linear Instability of a Highly Shear-Thinning Fluid in Channel Flow
}

\author{
Helen J. Wilson ${ }^{1}$, Vivien Loridan \\ Mathematics Department, University College London, Gower Street, London WC1E \\ $6 B T, U K$
}

\begin{abstract}
We study pressure-driven channel flow of a simple viscoelastic fluid whose elastic modulus and relaxation time are both power-law functions of shearrate. We find that a known linear instability for the case of constant elastic modulus [1] persists and indeed becomes more dangerous when the elastic modulus is allowed to vary. The most unstable scenario is a highly shearthinning relaxation time with a slightly shear-thinning elastic modulus, and typical unstable perturbations have a wavelength comparable with the channel width. Inertia is mildly destabilising.

We compare with microchannel experiments [2], and find qualitative agreement on the critical flow rate for instability; however, because of the artificial nature of the power-law viscosity, we have excluded the sinuous modes of instability which are seen in experiment.
\end{abstract}

Keywords: Channel flow, Instability, Shear-thinning, Power-law, elastic modulus, Elastic instability, Linear instability, Supercritical

\section{Introduction}

It is well known [3] that viscoelastic fluids can exhibit instabilities not seen in their Newtonian counterparts. Where such an instability persists in the absence of inertia, it is termed an elastic instability.

Perhaps the most well-understood elastic instability is the curved streamline instability discovered by Larson, Shaqfeh \& Muller [4] and elucidated by

\footnotetext{
${ }^{1}$ Email: helen.wilson@ucl.ac.uk. Tel: +44 (0)20 7679 1302. Fax: +44 (0)20 73835519.
} 
Pakdel \& McKinley [5]. Here the first normal stress difference interacts with curvature of the streamlines to drive an instability.

Another broad category of elastic instabilities is interfacial instabilities. A jump in material properties across an interface can trigger instability of the interface: either through a long-wave mechanism based on the tilting of the interface [6] or in some cases [7] by a mechanism that remains obscure (and persists even when surface tension holds the interface flat) but nonetheless depends critically on the presence of the interface.

A third category is shear-banding instabilities: a fluid whose constitutive curve is non-monotonic may spontaneously form bands of different shear stress (in a rate-controlled scenario) or different shear rate (in a stresscontrolled scenario) [8]. This seemingly unphysical behaviour does seem to occur for real physical systems [9] and has been the focus of much recent work [10].

However, recently published experiments by Bodiguel et al. [2] have found evidence of an elastic instability, occurring at a reproducible critical flow rate, in a flow having neither curved streamlines, nor an interface, nor any evidence of shear-banding. There is, to our knowledge, only one theoretical prediction of such an instability, in a study by Wilson \& Rallison [1]. In this paper we extend that analysis to a constitutive model which can match the rheometry of the fluid used in experiments. We find an instability whose critical flowrate is reasonably close to that seen in the experiments; but there are limitations to our model.

In section 2 we introduce our constitutive model and show its behaviour in simple shear flow; in section 3 we carry out a linear stability analysis for channel flow of this new fluid. In section 4 we present the results of our study, including the dependence of the instability on fluid parameters, on inertia and on perturbation wavenumber. In section 5 we make a detailed comparison with the experimental results published in [2]; finally in section 6 we draw our overall conclusions.

\section{Model Fluid}

Our model fluid is chosen with three principles in mind. It needs to match the rheometry of the experiments we wish to replicate [2]; some limit of it needs to match the existing theory [1]; and it is desirable for it to have at least a semi-physical microscopic derivation. 
In the experiments, the fluid is highly shear-thinning. The rheometry shows that both the viscosity and first normal stress difference are reasonably well fit with a simple power law over a good range of shear rates. Thus we need a viscoelastic model whose parameters can vary with shear rate.

Our previous theory [1] used a special case of the White-Metzner model whose relaxation time had power-law dependence on shear rate but whose modulus was independent of flow. We need to extend this fluid to allow a wider range of rheology in the fluid.

All White-Metzner style models are simply phenomenological extensions of the UCM model; UCM, on the other hand, does have a physical derivation as the polymer stress contribution of a dilute solution of Hookean dumbbells (see, for example [11]). The model we will use in this paper comes from a semi-physical extension of the UCM derivation, which is to allow the spring constant and the solvent viscosity to vary with the background shear rate (but without a kinetic theory to explain the behaviour of these two parameters). The derivation produces the following constitutive equation for the extrastress tensor $\underline{\underline{\tau}}$ :

$$
\underline{\underline{\tau}}=G(\dot{\gamma}) \underline{\underline{A}} \quad \underline{\underline{A}}=-\frac{1}{\lambda(\dot{\gamma})}(\underline{\underline{A}}-\underline{\underline{I}})
$$

in which the rheological functions $G$ (shear modulus) and $\lambda$ (relaxation time) depend on the instantaneous shear rate $\dot{\gamma}$, and $\underline{\nabla}$ is the upper-convected derivative, defined below in equation (6).

This is not equivalent to the standard White-Metzner model, which is given by the following equation:

$$
\underline{\underline{\tau}}+\lambda(\dot{\gamma}) \stackrel{\nabla}{\underline{\tau}}=\eta(\dot{\gamma})\left(\underline{\nabla u}+\underline{\nabla u}{ }^{\top}\right)
$$

in which $\eta(\dot{\gamma})$ is the shear-rate dependent viscosity and $\underline{u}$ the fluid velocity; however, in the special case $\eta(\dot{\gamma})=G \lambda(\dot{\gamma})$ for constant $G$, the two models both reduce to the form considered in [1].

\subsection{Governing equations}

The full governing equations for our incompressible fluid (in the absence of external body forces such as gravity) are:

$$
\begin{gathered}
\underline{\nabla} \cdot \underline{u}=0 \\
\rho\left(\frac{\partial \underline{u}}{\partial t}+\underline{u} \cdot \underline{\nabla u}\right)=\underline{\nabla} \cdot \underline{\sigma}
\end{gathered}
$$




$$
\begin{gathered}
\underline{\underline{\sigma}}=-p \underline{\underline{I}}+G \underline{\underline{A}} \\
\underline{\underline{A}}=-\frac{1}{\lambda}(\underline{\underline{A}}-\underline{\underline{I}}) \\
\underline{\underline{A}} \equiv \frac{\partial}{\partial t} \underline{\underline{A}}+\underline{\underline{u}} \cdot \underline{\underline{\nabla}}-(\underline{\underline{ }} \underline{\underline{A}})^{\top} \cdot \underline{\underline{A}}-\underline{\underline{A}} \cdot \underline{\nabla u} .
\end{gathered}
$$

Here $\underline{u}$ is the fluid velocity, $\rho$ its density, $\underline{\underline{\sigma}}$ the total stress tensor, $p$ pressure, $\underline{A}$ the conformation tensor, $G$ the elastic modulus and $\lambda$ the relaxation time. $\underline{\bar{I}}$ is the identity tensor (or unit matrix).

The parameters $\lambda$ and $G$ depend on the shear rate $\dot{\gamma}$, defined as an invariant of the rate-of-strain tensor $\underline{\underline{E}}$ as follows:

$$
\dot{\gamma}=\sqrt{2 \underline{\underline{E}}: \underline{\underline{E}}} \text { where } \underline{\underline{E}}=\frac{1}{2}\left(\underline{\nabla u}+\underline{\nabla u}{ }^{\top}\right) \text {. }
$$

\subsection{Rheometry}

We use cartesian coordinates $(x, y)$. In a simple steady shear flow $\underline{u}=$ $\dot{\gamma} y \underline{e}_{x}$ the fluid stress is

$$
\underline{\underline{\sigma}}=\left(\begin{array}{cc}
-p_{0}+G+2 G \lambda^{2} \dot{\gamma}^{2} & G \lambda \dot{\gamma} \\
G \lambda \dot{\gamma} & -p_{0}+G
\end{array}\right),
$$

which gives the two viscometric functions:

$$
\eta \equiv \frac{\sigma_{12}}{\dot{\gamma}}=G \lambda \quad \Psi_{1} \equiv \frac{\sigma_{11}-\sigma_{22}}{\dot{\gamma}^{2}}=2 G \lambda^{2}
$$

As we will see in section 5, for the fluid used in the experiments it is reasonable, over a range of shear rates, to approximate both $\eta$ and $\Psi_{1}$ with power-law functions of $\dot{\gamma}$ of the form $A \dot{\gamma}^{n}$. This allows us to restrict our model to power-law behaviour for the functions $G(\dot{\gamma})$ and $\lambda(\dot{\gamma})$ :

$$
G=G_{M} \dot{\gamma}^{m-n} \quad \lambda=K_{M} \dot{\gamma}^{n-1}
$$

where the indices $m$ and $n$ are chosen so that the definition of $\lambda$ matches that used in [1], and the shear stress has the simple scaling

$$
\sigma_{12} \sim G_{M} K_{M} \dot{\gamma}^{m} .
$$




\section{Stability theory}

We now consider two-dimensional channel flow of a fluid satisfying equations (2-7) along with the scaling laws of equation (8). The channel, of infinite extent in the $x$-direction, has half-height $L$ (in the $y$-direction) and the flow is driven by a pressure gradient $\mathcal{P}$ in the $x$-direction.

\subsection{Steady channel flow}

If we assume a steady, unidirectional flow profile $\underline{u}=U(y) \underline{e}_{x}$, satisfying a no-slip condition at $y= \pm L$, we obtain the following solution:

$$
\begin{gathered}
U(y)=\left(\frac{\mathcal{P}}{G_{M} K_{M}}\right)^{1 / m}\left(\frac{m}{m+1}\right)\left(L^{(m+1) / m}-|y|^{(m+1) / m}\right) \\
\dot{\gamma}=\left|U^{\prime}\right|=\left(\frac{\mathcal{P}|y|}{G_{M} K_{M}}\right)^{1 / m} .
\end{gathered}
$$

\subsection{Dimensionless form}

We now convert to dimensionless quantities. We scale lengths with $L$, the channel half-width; times with the average shear rate $U_{0} / L$; and stresses with a typical shear stress, which is the fluid shear stress $\sigma_{12}$ at the average shear rate: $G_{M} K_{M}\left(U_{0} / L\right)^{m}$.

Denoting scaled quantities with a tilde, our new governing equations become:

$$
\begin{array}{ll}
\underline{\nabla} \cdot \underline{\tilde{u}}=0 & R e\left(\frac{\partial \underline{\tilde{u}}}{\partial t}+\underline{\tilde{u}} \cdot \underline{\nabla \tilde{u}}\right)=\underline{\nabla} \cdot \underline{\underline{\tilde{\sigma}}} \\
\underline{\underline{\sigma}}=-\tilde{p} \underline{\underline{I}}+\frac{C}{\mathcal{W}} \underline{\underline{A}} \quad \underline{\underline{A}}=-\frac{1}{\mathcal{W}}(\underline{\underline{A}}-\underline{\underline{I}})
\end{array}
$$

along with the definitions of $\underline{\nabla}$ and $\dot{\gamma}$ which are unchanged (save for the addition of tildes) from their original form in equations (6) and (7). (Note that $\underline{A}$ was dimensionless in the original equations and has not been scaled.)

We have introduced the Reynolds number (based on the shear viscosity at the average shear rate)

$$
R e=\frac{\rho U_{0} L}{G_{M} K_{M}\left(U_{0} / L\right)^{m-1}}
$$

and the new viscometric functions

$$
C=\dot{\gamma}^{m-1} \quad \mathcal{W}=W \dot{\gamma}^{n-1}
$$

where $W=K_{M}\left(U_{0} / L\right)^{n}$ as in [1]. We will suppress tildes henceforth. 


\subsection{Base state}

To carry out a linear stability analysis, we begin by calculating a base state whose stability is to be studied. This is simply the steady flow, invariant in the $x$-direction, which is the simplest channel flow solution to the governing equations.

In dimensionless form, the velocity profile is

$$
\underline{u}=(U(y), 0) \quad U=1-|y|^{(m+1) / m} \quad \dot{\gamma}_{0}=\left|U^{\prime}\right|=\frac{(m+1)}{m}|y|^{1 / m} .
$$

The base state viscometric functions are

$$
\begin{aligned}
C_{0} & =\dot{\gamma}_{0}^{m-1}=\left[\frac{(m+1)}{m}\right]^{m-1}|y|^{(m-1) / m} \\
\mathcal{W}_{0} & =W \dot{\gamma}_{0}^{n-1}=W\left[\frac{(m+1)}{m}\right]^{n-1}|y|^{(n-1) / m}
\end{aligned}
$$

so the pressure, conformation tensor and stress tensor become, respectively,

$$
\begin{gathered}
P_{0}=P_{\infty}+\frac{C_{0}}{\mathcal{W}_{0}}-\mathcal{P} x \\
\underline{\underline{A}} \equiv\left(\begin{array}{cc}
A_{11} & A_{12} \\
A_{21} & A_{22}
\end{array}\right)=\left(\begin{array}{cc}
1+2 W^{2}(\mathcal{P} y)^{2 n / m} & -W(\mathcal{P} y)^{n / m} \\
-W(\mathcal{P} y)^{n / m} & 1
\end{array}\right) \\
\underline{\underline{\sigma}} \equiv\left(\begin{array}{cc}
\Sigma_{11} & \Sigma_{12} \\
\Sigma_{21} & \Sigma_{22}
\end{array}\right)=\left(\begin{array}{cc}
-P_{\infty}+\mathcal{P} x+2 W \mathcal{P}^{n}|y|^{(m+n) / m} & -\mathcal{P} y \\
-\mathcal{P} y & -P_{\infty}+\mathcal{P} x
\end{array}\right)
\end{gathered}
$$

Note that $\mathcal{P}$ now represents the dimensionless pressure gradient $\mathcal{P}=[(m+1) / m]^{m}$.

\subsection{Perturbation flow}

We now add a spatially periodic perturbation to our base flow, so that all quantities are modified by an infinitesimally small change:

$$
\begin{aligned}
\underline{u} & =(U+u \mathcal{E} v \mathcal{E}) \\
\dot{\gamma} & =\dot{\gamma}_{0}+\dot{\gamma}_{1} \mathcal{E} \\
C & =C_{0}+c \mathcal{E} \\
\mathcal{W} & =\mathcal{W}_{0}+w \mathcal{E}
\end{aligned}
$$




$$
\begin{aligned}
& \underline{\underline{A}}=\left(\begin{array}{cc}
A_{11}+a_{11} \mathcal{E} & A_{12}+a_{12} \mathcal{E} \\
A_{12}+a_{12} \mathcal{E} & A_{22}+a_{22} \mathcal{E}
\end{array}\right) \\
& \underline{\underline{\sigma}}=\left(\begin{array}{ll}
\Sigma_{11}+\sigma_{11} \mathcal{E} & \Sigma_{12}+\sigma_{12} \mathcal{E} \\
\Sigma_{12}+\sigma_{12} \mathcal{E} & \Sigma_{22}+\sigma_{22} \mathcal{E}
\end{array}\right)
\end{aligned}
$$

and so on, in which we are considering a single Fourier mode:

$$
\mathcal{E}=\varepsilon \exp [i k x-i \omega t]
$$

The conservation of mass equation (2) becomes

$$
i k u+\mathrm{d} v / \mathrm{d} y=0
$$

which we satisfy automatically by introducing a streamfunction $\psi$ and setting

$$
u=\mathrm{d} \psi / \mathrm{d} y \quad v=-i k \psi .
$$

We now introduce the notation $D$ to denote $\mathrm{d} / \mathrm{d} y$ for convenience. Substituting the perturbed forms into the governing equations and discarding terms of order $\varepsilon^{2}$ we obtain the following linear system:

$$
\begin{aligned}
& i k \sigma_{11}+D \sigma_{12}=\operatorname{Re}(-i \omega D \psi-i k \psi D U+i k U D \psi) \\
& i k \sigma_{12}+D \sigma_{22}=\operatorname{Re}\left(-k \omega \psi+k^{2} U \psi\right) \\
& \sigma_{i j}=-p \delta_{i j}+\frac{C}{\mathcal{W}} a_{i j}+\left(\frac{c}{\mathcal{W}}-\frac{C w}{\mathcal{W}^{2}}\right) A_{i j} \\
& \left(-i \omega+i k U+\mathcal{W}^{-1}\right) a_{11}=i k \psi D A_{11}+2 a_{12} D U+2 A_{11} i k D \psi \\
& +2 A_{12} D^{2} \psi+\frac{w}{\mathcal{W}^{2}}\left(A_{11}-1\right) \\
& \left(-i \omega+i k U+\mathcal{W}^{-1}\right) a_{12}=i k \psi D A_{12}+a_{22} D U+A_{11} k^{2} \psi \\
& +D^{2} \psi+\frac{w}{\mathcal{W}^{2}} A_{12} \\
& \left(-i \omega+i k U+\mathcal{W}^{-1}\right) a_{22}=2 A_{12} k^{2} \psi-2 i k D \psi
\end{aligned}
$$

in which the perturbations to the viscometric functions are

$$
\begin{aligned}
c & =(m-1) \dot{\gamma}_{0}^{m-2} \dot{\gamma}_{1} \\
w & =W(n-1) \dot{\gamma}_{0}^{n-2} \dot{\gamma}_{1}
\end{aligned}
$$


and the perturbation shear rate,

$$
\dot{\gamma}_{1}=-\left(D^{2}+k^{2}\right) \psi
$$

The system defined by equations (30-38) can be combined (forming the vorticity equation) to give a fourth-order ODE in $\psi$. The boundary conditions are simply conditions of no flow $(\psi=D \psi=0)$ on the boundaries $y= \pm 1$.

The system is now governed by five dimensionless parameters: the fluid parameters $m$ and $n$, the flow rate parameters $W$ and Re, and the wavenumber $k$.

We solve the system given by equations (30-38) numerically, using the shooting method of Ho \& Denn [12] for a given real value of the wavenumber $k$. We use numerical parameter continuation to give initial guesses as we move away from the known results given in [1].

\subsection{Centreline singularity}

As described in [1], there is a complication at the centreline $y=0$. Because $\dot{\gamma}_{0}=0$ at that point, the viscometric functions become singular. This is clearly unphysical, and is an artefact of assuming a power-law even for very low shear-rates; however, if we limit ourselves to varicose perturbations (for which the streamfunction $\psi$ is an odd function of $y$ ) for which $\dot{\gamma}_{1}=0$ at the centreline, we can draw sensible conclusions without having to extend the fluid model. This issue is discussed in more detail on pages 79-80 of [1].

\section{Results}

We know that our model fluid is capable of predicting instability, because the case $n=m$ has been studied before [1]. In this section we show how the instability depends on the key flow parameters.

\subsection{Extremes of wavelength}

As for the previously-studied case $m=n$, there is no neutrally-stable eigenvalue as $k \rightarrow 0$ (as there would be for an interfacial instability [6]); rather, all modes are stable in the long-wave limit. However, while the calculation to leading and first orders in $k$ was accessible analytically in the case $m=n$ (involving nothing more difficult than an asymptotic expansion, and integration of functions of the form $y^{a}$ ), in the new scenario even the 
leading-order integration involves the hypergeometric function ${ }_{2} F_{1}$ and the resulting dispersion relation:

$$
\begin{aligned}
\frac{\omega}{(n+2 m)}+ & \frac{i n}{W^{\prime}[n+(m-n) i \omega](1+2 m)} \\
& +\sum_{j=2}^{\infty}\left[\omega\left(\frac{1}{j}-\frac{1}{(j-1)}\right)+\frac{i n}{(m-n)} \frac{1}{(j-1)}\right] \times \\
& \frac{1}{(n+2 m+j(1-n))}\left(\frac{(m-n)}{W^{\prime}[n+(m-n) i \omega]}\right)^{j}=0
\end{aligned}
$$

(in which we have set $W^{\prime}=W[(m+1) / m]^{(n-1)}$ ) cannot be solved algebraically for $\omega$.

For short waves (the limit $k \rightarrow \infty$ ), the situation is very similar to that described in [1]. Provided that $k$ is sufficiently large that the wavelength is shorter than the size of the boundary layer over which the shear rate changes near the wall, any eigenfunction becomes localised in a region of size $k^{-1}$. Considering only those which localise close to the wall, the important timescale relates to the wall shear rate $\dot{\gamma}_{w}=(m+1) / m$ and so, although the resulting scaled system can only be solved numerically, in the limit of small $m$, the quantity $\omega \dot{\gamma}_{w}^{-1}$ must remain finite so any instability must scale as $\omega \sim \dot{\gamma}_{w} \sim m^{-1}$ for small $m$. This eigenvalue does not depend on $k$ in the limit $k \rightarrow \infty$, reflecting the underlying stability properties of unbounded steady shear flow past a single wall at a shear rate $\dot{\gamma}_{w}$.

\subsection{Continuous spectrum}

As with all stability problems of this type, in addition to the discrete eigenvalues (some of which may be unstable) there is a continuous spectrum representing the local fluid response to a highly localised perturbation. It is characterised by a singularity in the highest derivative in the governing equations (at a specific cross-channel location $y_{0}$ ), and the characteristic perturbation associated with it is zero everywhere except at $y=y_{0}$. In this case the singularity occurs when the coefficient

$$
\left(-i \omega+i k U+\mathcal{W}^{-1}\right)
$$

in equations (33-35) vanishes, giving the eigenvalue

$$
\omega=k\left(1-y_{0}^{(m+1) / m}\right)-\frac{i}{W}\left[\frac{(m+1)}{m}\right]^{1-n} y_{0}^{(1-n) / m}
$$




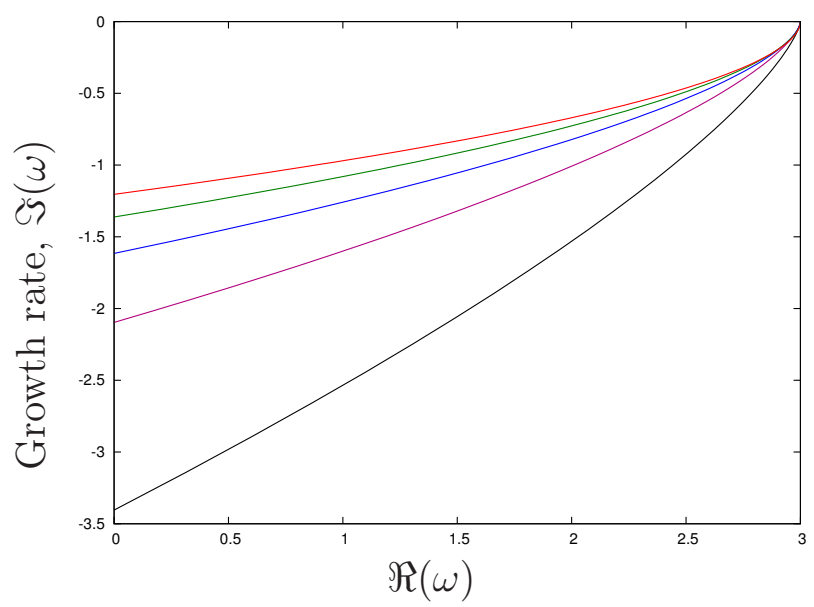

Figure 1: The continuous spectrum at $n=0.2, k=3, W=2$. From top to bottom: $m=0.5,0.4,0.3,0.2,0.1$.

for any value $0 \leq y_{0} \leq 1$. Figure 1 gives sample continuous spectra for different values of $m$, all at the exemplar value $n=0.2$. The curves are similar in shape for other values of the physical parameters.

\subsection{Effect of inertia}

We take three set of parameters $\{m, n, W\}$ for which we find a purely elastic instability, and in each case choose a wavenumber $k$ near which the growth rate peaks at $R e=0$. Then, fixing the wavenumber along with the other parameters, we add inertia by increasing Re. The results are plotted in figure 2; we can see that weak inertia is destabilising in all cases. This is in contrast to curved-streamline elastic instabilities, in which inertia acts to oppose the elastic forces and can be stabilising.

\subsection{Effect of variable modulus}

The case of constant shear modulus, first studied in [1], is given by $m=n$. When $m$ is free to take other values, we find typically that values $m<n$ (shear-thinning modulus) are more susceptible to instability than values $m>$ $n$ (shear-thickening modulus). However, the picture is not simple: in figure 3 we plot the growth rate against the modulus $m$ for indicative parameter values $k=3, W=2, R e=0$ and three chosen values of the relaxation time power-law, $n=0.1,0.2$ and 0.3 . These are sufficiently low that previous work did identify an instability at $m=n$; for higher values of $n$, the case $m=n$ is linearly stable. 


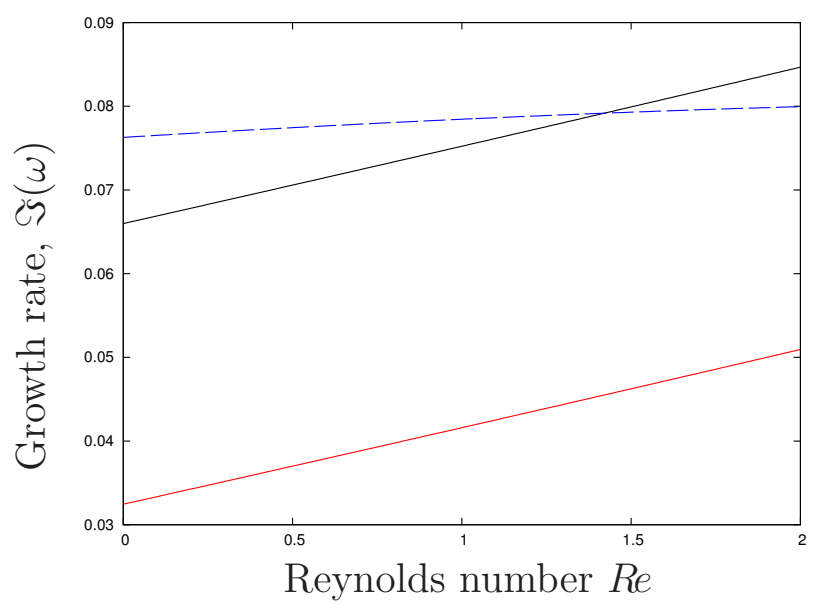

Figure 2: Effect of inertia on growth rate. Case 1 (top solid line): $m=0.2, n=0.4$, $W=2, k=4.2$. Case 2 (bottom solid line): $m=0.2, n=0.3, W=2, k=3.5$. Case 3 (dashed line): $m=0.2, n=0.1, W=3, k=3.75$ (here $G$ shear-thickens, in contrast to cases 1 and 2).

We see that, for a given value of $n$, the most unstable scenario at this wavenumber and flow rate is given by $m<n$ (a shear-thinning elastic modulus) but not by the limit $m \rightarrow 0$. We cannot access the limit of very low $m$ numerically because of the small size of the boundary layer in the base flow, which causes the perturbation equations to be very stiff; but from our results for $m>0.05$ it is clear that the limit $m \rightarrow 0$ will be stable at this wavenumber. This is in contrast to the short-wave limit where, as discussed in section 4.1, instability is expected for very small $m$.

In all cases presented in figure 3 the flow is stable if $m$ is greater than around 0.2 . There is a distinctive double-peak structure at $n=0.2$ and $n=0.3$, which is not present at the much more unstable $n=0.1$.

In the lower part of figure 3 we zoom in on three instances of odd-looking behaviour in the dependence of the eigenvalue $\omega$ on $m$, taking as our example the middle case $n=0.2$. First at $m=0.2$ (figure $3(\mathrm{a})$ ) there is a local blip on the curve, associated with a rapid change in slope. This occurs generically at $m=n$, and can just be seen in the main figure at $m=0.1$ for the curve $n=0.1$. The other two features occur in the stable portion of the curve. At $m=0.3175$ (figure $3(\mathrm{~b})$ ), the root jumps. This is not a generic feature, and does not occur at a fixed value of $n$ or indeed for all parameter values; we have observed several instances of it, always within the stable region. 


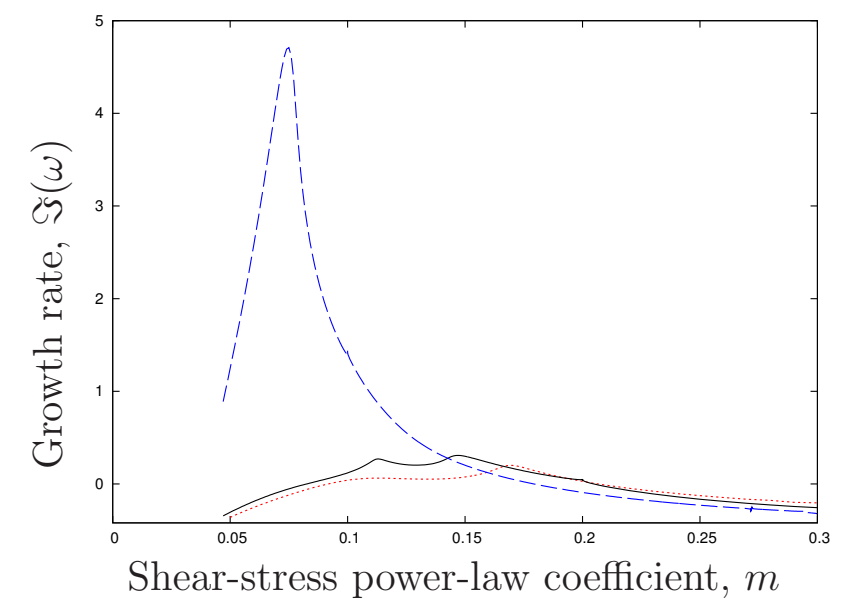

(a)

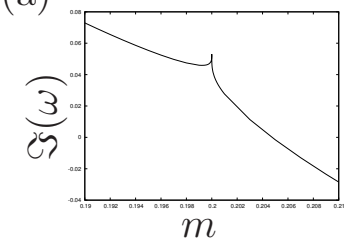

(b)

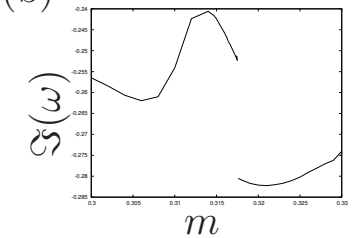

(c)

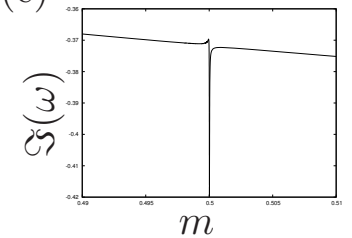

Figure 3: Plot of growth rate against the power-law $m$ which governs the shear stress. The most unstable mode is shown, and we have fixed $R e=0, W=2, k=3$ and $n=0.1$ (dashed line), $n=0.2$ (solid line) or $n=0.3$ (dotted line). The case $m=n$, in which the shear modulus is constant, corresponds to that studied in [1]. Parts (a), (b) and (c) are small regions of the wider curve for $n=0.2$. 
Finally, at $m=0.5$ (figure 3(c)), the growth rate suddenly changes markedly (but continuously) only to return sharply back to a value similar to the value before the change. This near-discontinuity always occurs around the point $m=0.5$ independent of $n$; again, the flow is stable at this point.

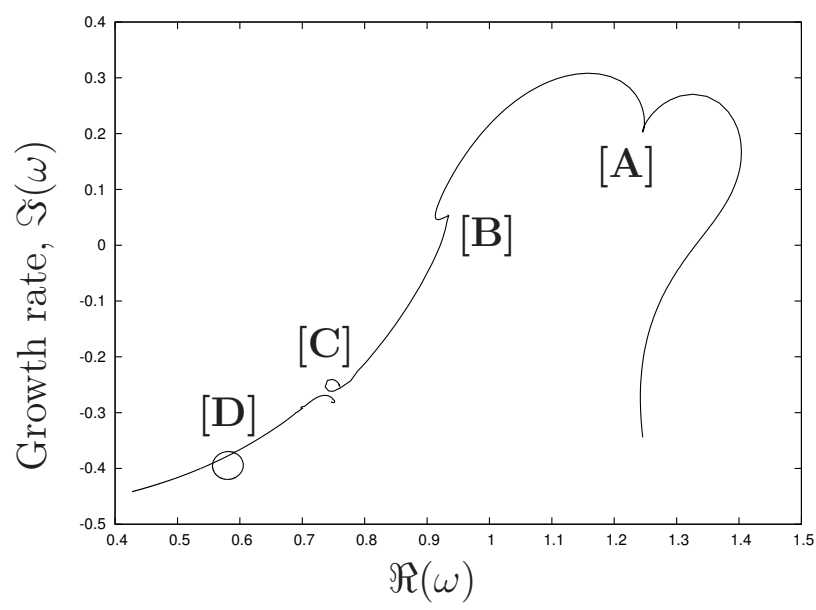

Figure 4: Plot of the eigenvalue $\omega$ in the complex plane as $m$ varies with $R e=0, W=2$, $k=3$ and $n=0.2$.

In figure 4 we show the behaviour of the most unstable eigenvalue in the complex $\omega$-plane, at the same parameters as the solid curve in figure 3, i.e. $k=3, W=2, n=0.2, R e=0$ and varying $m$. Values for low $m$ are at the right hand side; the apparent "cusp" at $[\mathbf{A}]$ is merely the local minimum in growth rate around $m \approx 0.15$. The kink at $[\mathrm{B}]$ is the slope discontinuity at $m=n$. The curve is genuinely discontinuous at $m=0.3175[\mathbf{C}]$. Finally, the feature at $m=0.5$ is the loop at $[\mathbf{D}]$. Despite appearances, there is just one, continuous, curve here: as $m$ increases the eigenvalue approaches the loop from the right, traverses the loop anticlockwise as $m$ decreases from 0.501 to 0.499 , and then continues along the "original" curve.

\subsection{Stability boundary}

For a given set of fluid parameters, the most important question is: at what flow rate will our flow become unstable? The details of the instability the most dangerous wavenumber, and the form of the unstable perturbation - come second.

In figure 5 we address this question for fixed $m=0.2$ and $R e=0$ and a range of values of the timescale power-law, $n$. 


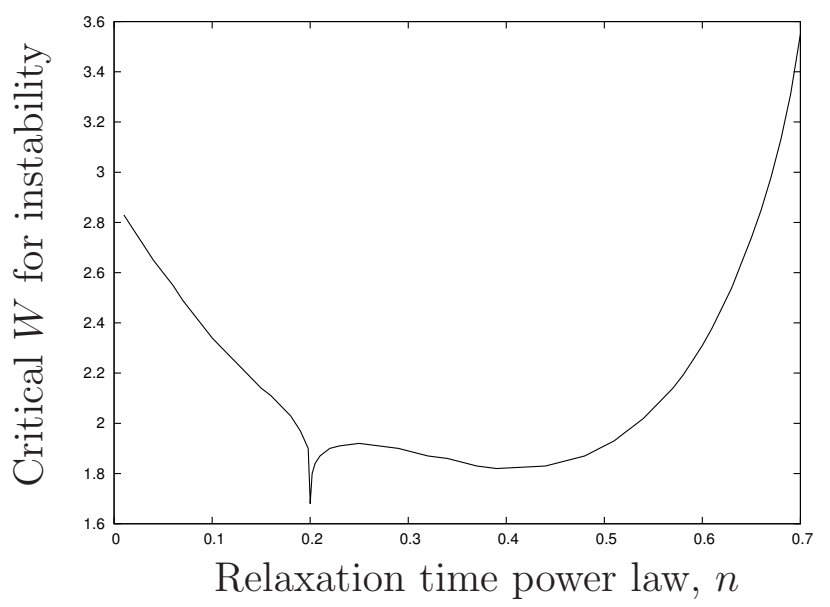

Figure 5: Plot of the critical Weissenberg number $W_{\text {crit }}$ against the power law $n$ governing the relaxation time, with $R e=0$ and $m=0.2$.

We see that the critical Weissenberg number is lowest at $n=0.2$, the case previously studied [1]; the case $m=n$ does appear to be weakly singular, as suggested by the "kink" at $m=n$ in figure 3 . There is also a local minimum around $n=0.4$ : this coincides with our observation in section 4.4 that a weakly shear-thinning modulus $(m<n)$ is a particularly unstable scenario. By chance, this also roughly matches the fluid used in the experiments of [2], as we will see in section 5 . As $n$ becomes very small or very large, the critical Weissenberg number increases.

It is perhaps surprising that the instability persists up to such large values of $n$ : we have found it up to $n=0.82$, where the critical Weissenberg number is over 15 , whereas the special case $m=n$ is stable for all $n>0.3$. In all cases featured in figure 5 the instability seen at $W_{\text {crit }}$ has a wavenumber $1.9 \leq k \leq 4.6$; for all but the lowest values of $n$ (where $k \approx 4.5$ ) and the small region around $n=0.2$ (where $k$ drops sharply to its minimum value of 1.9) the most dangerous waves have $3 \leq k \leq 4$, i.e. a wavelength of the same order as the channel width.

\subsection{Dependence on wavenumber}

In figure 6(a) we show plots of growth rate against wavenumber for two specific cases, corresponding to the two local minima of figure 5. For completeness, the corresponding real parts of the eigenvalue are plotted in figure $6(\mathrm{~b})$. 
(a)

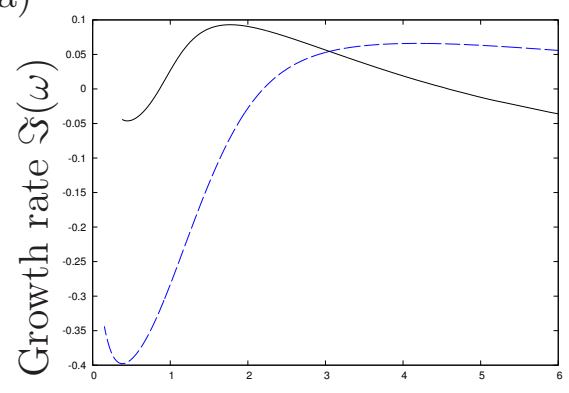

Wavenumber, $k$ (b)

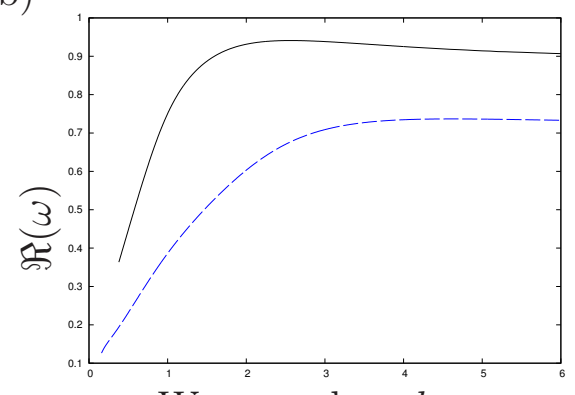

Wavenumber, $k$

Figure 6: Plots of the complex eigenvalue $\omega$ against wavenumber for the most unstable mode. (a) Growth rate, given by the imaginary part of $\omega$; (b) real part of $\omega$. Solid curves: $m=0.2$; dashed curves: $m=0.4$. The other dimensionless parameters are $n=0.2, W=2$ and $R e=0$; thus the curve for $m=0.2$ corresponds to [1], in which $m=n$.

We see that, in each case, very long waves are stable (as expected) and the most unstable wavenumber is finite: at $n=0.2$ we have $k_{\max } \approx 1.8$ giving a wavelength $3.5 L$, while at $n=0.4$ the wavenumber is higher, $k_{\max } \approx 4.2$ giving shorter waves of wavelength $1.5 \mathrm{~L}$.

Considering short waves, in each case the eigenvalue remains finite as $k \rightarrow \infty$, as predicted in section 4.1 ; in fact the behaviour for very short waves is well approximated with the asymptotic form

$$
\Im(\omega) \sim \omega_{\infty}+k^{-1} \beta
$$

At $n=0.2$ we find $\omega_{\infty}=-0.0168$ and $\beta=0.8$, while at $n=0.4$ we have $\omega_{\infty}=-0.02$ and $\beta=0.48$. In both cases, very short waves are stable; the value for $n=0.2$ matches the short-wave limit calculated in [1]. In each case $\Re(\omega)$ remains finite as $k \rightarrow \infty$, indicating that short wave perturbations become localised in the wall region (rather than localising at a cross-channel position $\bar{y}$ and convecting with the flow at velocity $U(\bar{y})$, which would yield $\Re(\omega) \sim k U(\bar{y})$ as $k \rightarrow \infty)$.

The new mode of instability $(n=0.2, m=0.4)$ is unstable to a much wider range of wavenumbers than the previously known instability having the same relaxation time: waves having $2.2<k<24$ are now unstable as opposed to the previous range $0.87<k<4.5$, even though increasing $m$ (which is the only change here) causes the viscosity to be less shear-thinning than in the original constitutive model. 


\section{Experiments}

In this section we briefly describe the experiments published in [2] and compare the instability seen there with our theoretical prediction.

\subsection{Experimental setup and parameters}

These experiments are described in full by Bodiguel et al. [2]. Briefly, an aqueous solution of polyacrylamide (density $\rho=10^{3} \mathrm{~kg} \mathrm{~m}^{-3}$ ) was driven through a microchannel (of half-width $L=76 \mu \mathrm{m}$ or $85 \mu \mathrm{m}$ ). The velocity field was measured by PIV.

Using a sanded cone-and-plate rheometer in controlled shear-rate mode, the fluid rheology is reported as

$$
\sigma_{12}=3.73 \dot{\gamma}^{0.21} \mathrm{~Pa} \quad \frac{\sigma_{11}-\sigma_{22}}{2 \sigma_{12}}=3.63 \dot{\gamma}^{0.43},
$$

which maps onto our fluid model (1) if we set $G_{M}=1.03 \mathrm{Pas}^{-0.22}, K_{M}=$ $3.63 \mathrm{~s}^{0.43}, m=0.21$ and $n=0.43$. If the centreline velocity is $U_{0}=\hat{u} \mathrm{~mm} \mathrm{~s}^{-1}$ we can deduce the dimensionless parameters shown in table 1.

\begin{tabular}{clc}
\hline Parameter & $L=85 \mu \mathrm{m}$ & $L=76 \mu \mathrm{m}$ \\
\hline$m$ & 0.21 & 0.21 \\
$n$ & 0.43 & 0.43 \\
$W$ & $10.5 \hat{u}^{0.43}$ & $11.0 \hat{u}^{0.43}$ \\
$k$ & any & any \\
$R e$ & $1.59 \times 10^{-4} \hat{u}^{0.79}$ & $1.56 \times 10^{-4} \hat{u}^{0.79}$ \\
\hline
\end{tabular}

Table 1: Dimensionless parameters calculated for the experiments of [2] at a centreline velocity of $\hat{u} \mathrm{~mm} \mathrm{~s}^{-1}$.

\subsection{Comparison of experiments and theory}

The key observation in these experiments is the appearance of large oscillations in the velocity field at a highly-reproducible value of the wall shear stress

$$
\sigma_{w}^{\text {crit }}=4.7 \pm 0.2 \mathrm{~Pa},
$$

which corresponds to a critical Weissenberg number of

$$
W^{\text {crit }}=2.75 \pm 0.25 \text {. }
$$


For the fluid used in these experiments, our calculations predict instability at $W \approx 1.8$. While this is not in quantitative agreement with the experimental observations, the discrepancy is not huge and it is likely that the same mechanism is driving both the theoretical and experimental instabilities.

We note in passing that, as expected for such small channels, inertia can indeed be neglected in these experiments: the maximum Reynolds number is roughly $1.6 \times 10^{-5}$.

In the experiments, it is possible to extract a period of oscillations from the PIV data. This value should be treated with some caution: these are observations of a fully developed unsteady flow which is no longer in the linear régime and the frequency need not match exactly the real part of the most linearly unstable eigenvalue. However, it is nonetheless informative to compare these observations with the linear theory.

The period $T$ of oscillations for a chosen material particle in our theory depends on the particle's average transverse position in the channel, $y$ :

$$
T=\frac{2 \pi L}{|\omega-k U(y)| U_{0}} .
$$

However, in the experiments, a single period of oscillation is reported. This is because across the majority of the channel the bulk velocity is close to the centreline velocity (the difference in velocity is less than $10 \%$ in well over half of the channel). The subtle differences in observed period are largely manifest in particles close to the wall, whose oscillations are constrained by the wall and less easy to observe.

The period observed in experiments, in the $L=76 \mu \mathrm{m}$ channel at a wall shear stress of $\sigma_{w}=7.8 \mathrm{~Pa}$, is $1.2 \mathrm{~s}$. If we approximate $U(y)$ with 1 , the dimensionless centreline velocity, in the theoretical calculation, our prediction is $1.15 \mathrm{~s}$. Again, there is good agreement between the experimental observation and the theoretical prediction, indicating that we are seeing the correct instability mechanism even though the details of our constitutive equation are unlikely to be accurate.

It should be noted, also, that the oscillations seen in experiment are not damped near the centreline of the flow, which means our limitation to varicose mode perturbations does prevent us from making quantitatively accurate predictions.

In figure 7 we show the form of the unstable perturbation, at the most dangerous wavenumber for this fluid, at a flow rate of $W=2$ (just above criticality). There is a strong peak in $\psi$ (corresponding to a peak in the 
(a)

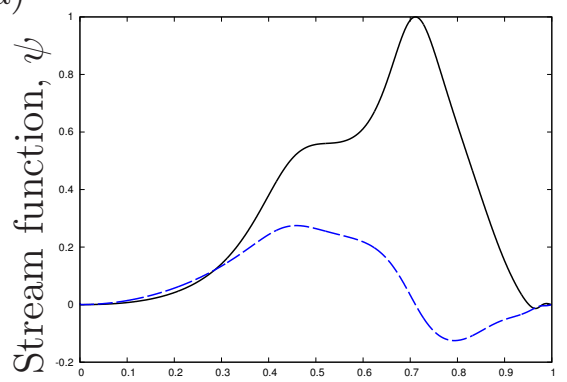

Cross-channel co-ordinate, $y$ (b)

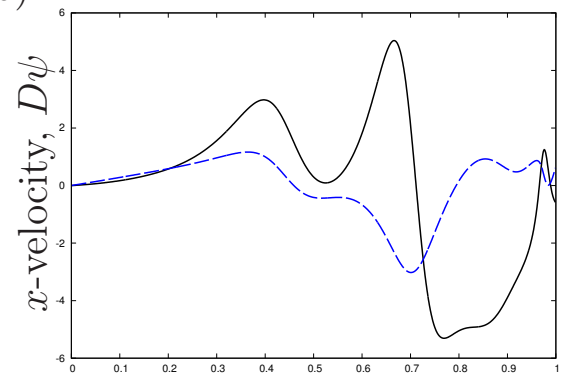

Cross-channel co-ordinate, $y$

Figure 7: Form of the complex streamfunction $\psi$ for the unstable perturbation (normalised so that the maximum value of $|\psi|$ is 1 ). In each graph, the solid curve is the real part and the dashed curve the imaginary part. Parameters $n=0.2, m=0.4, W=2, R e=0$, and the wavenumber $k=4.18$ of the most unstable perturbation for these flow parameters. (a) Streamfunction, $\psi$ : recall that the cross-channel velocity is $i k \psi$. (b) Derivative $D \psi$, equal to the velocity along the channel.

cross-channel perturbation velocity) at $y \approx 0.7$, which drives fluid into and out of the highly-sheared boundary layer. Perhaps more surprising is the double-peak in the velocity along the channel: the first of these occurs where $U=0.96$ and the second, $U=0.74$. The small artefacts in the graphs of $D \psi$ close to the wall $y=1$ are exactly that and should be discounted. It should be noted that, since $k=4.18$, the maximum velocities in the $x$-direction $(D \psi)$ and $y$-direction $(i k \psi)$ are of the same magnitude.

Finally, it is of interest to consider the extent to which the variation in the shear modulus and relaxation time is important. Thus, we consider fluids which best model the experiments while keeping either the shear modulus constant (in dimensional form, $G=G_{M}, m=n$ ) or the relaxation time constant (in dimensional form, $\lambda=K_{M}, n=1$ ). In each case we choose dimensional parameters such that the shear stress $\sigma_{12}=G_{M} K_{M} \dot{\gamma}^{m}$ matches the rheometry, and fix the constant value (whether relaxation time or shear modulus) using the value from our original power law and the average shear rate across the channel. The parameters used in this comparison are given in table 2 .

We find that the case of constant relaxation time shows no instability at all; in the case of constant modulus (as in [1]) there is instability at a critical Weissenberg number $W_{\text {crit }}=1.70$ to a perturbation of wavenumber $k=1.58$, which are both of the same order as the experimental observations; 


\begin{tabular}{lllll} 
& $m$ & $n$ & $G_{M}$ & $K_{M}$ \\
\hline Full fluid match & 0.21 & 0.43 & $1.03 \mathrm{~Pa} \mathrm{~s}^{-0.22}$ & $3.63 \mathrm{~s}^{0.43}$ \\
Constant relaxation time & 0.21 & 1.00 & $0.76 \mathrm{~Pa} \mathrm{~s}^{-0.79}$ & $4.93 \mathrm{~s}$ \\
Constant modulus & 0.21 & 0.21 & $1.16 \mathrm{~Pa}$ & $3.22 \mathrm{~s}^{0.21}$ \\
\hline
\end{tabular}

Table 2: Modelling parameters for the three different fits to the experiments of [2]: the best fit; a fit having a constant relaxation time; and a fit having constant shear modulus.

but the predicted period of oscillation is $T=209 \mathrm{~s}$, much longer than is seen in experiment.

\subsection{Effects of wall slip}

In the experiments a large slip velocity is observed at the channel wall, even for slow, stable flows. For the purposes of the stability analysis, we will assume that there is a uniform slip velocity $V_{s}$ which is constant in space and time, and that the perturbation flow does not involve any additional slip velocity. Thus the total velocity becomes

$$
\underline{u}=V_{s} \underline{e}_{x}+U(y) \underline{e}_{x}+\text { perturbation velocity }
$$

where $U(y)$ is the base flow calculated earlier. The only effect of this change is to effectively change the frame of reference of the whole flow: because of the unidirectional nature of the base flow, there are no inertial effects caused by the shift. The eigenvalue $\omega$ (relative to the original frame of reference) has identical growth properties to the original system, but is shifted by the real contribution $k V_{s}$. This shift causes the perturbation to translate downstream with the slip velocity.

With this (admittedly rather strong) assumption about the behaviour of the wall boundary conditions in the presence of slip, we are able to apply the theoretical results for growth or decay of instability without any effect of the wall slip.

\subsection{Observations of memory effects}

A further observation by Bodiguel et al. [2] is that the mean flow rate in the channel is increased following the onset of instability. This is an inherently nonlinear effect (by construction, the linear perturbation mode has zero flux at any time instant, and is also time-periodic) and as such we cannot capture it with linear stability theory. But there is a deeper problem here. 
Bodiguel et al. propose a mechanism for the enhanced flux via homogenisation. Essentially, it is argued that fluid which has low viscosity due to high shear rate near the wall is transported out into the channel by the perturbation flow, and carries its low viscosity with it. This fits quantitatively with the data, given a simple diffusion equation for the fluidity, with a diffusion length which is well modelled by the relaxation time multiplied by the RMS cross-channel velocity. The authors argue that this is the distance over which a polymer molecule will remember its conformation and therefore its material properties.

In our toy constitutive model, the material properties $G$ and $\lambda$ respond instantaneously to their local shear rate. Thus, even with a fully nonlinear calculation, we know that our model could not capture the enhanced flux via the mechanism discovered in experiment. A truly quantitative experimental prediction will need a model in which the material properties themselves have a relaxation time over which they respond to a change in shear rate. This could be captured, at its simplest (and in a physically justifiable way), by a structure factor, built up or destroyed by flow, on which the material properties depend.

\subsection{Other possible mechanisms}

The linear instability predicted by our theory is not the only possible explanation for the experimental observations. Before the fluid enters the channel, it will have travelled through a region of curved streamlines. The wellunderstood curved-streamline instability [5] could be triggered there (which would occur at a reproducible flow rate, since it is a supercritical instability) and simply advected into the channel, triggering either our instability or some nonlinear instability within the channel.

Equally, there is the possibility of non-modal growth [13, 14, 15]. Essentially, if the linear stability problem has close eigenvalues, we may see transient growth of disturbances even if the long-time behaviour (predicted by the sign of the eigenvalues) is stable. This transient growth can then trigger a nonlinear instability. It is not clear whether or not this transient growth (which certainly can occur in viscoelastic channel flows) would occur at a reproducible critical flow rate, but if so then it is another candidate explanation for the experimental observations. 


\section{Conclusions}

We have studied the stability properties of channel flow of a highly shearthinning viscoelastic fluid. The well-known mechanisms of curved streamlines, interfaces, or shear-banding do not apply here. We extend previous work [1] to allow the elastic modulus and relaxation time to shear-thin (or shear-thicken) independently of one another (previously the elastic modulus $G$ was constant). We find that a slightly shear-thinning elastic modulus has a strongly destabilising effect; that weak inertia is also destabilising (in constrast to the curved-streamline instability); and that the most unstable perturbation typically has wavelength comparable with the channel width.

We compare directly with the experiments of [2]. For the fluid used in those experiments, both long waves and short waves are stable, and a finite region of wavenumbers shows instability at a given flow rate. We have qualitative agreement with the experiments on critical flow rate and on the period of the oscillations.

The primary weakness of our theory is that we cannot capture sinuous perturbations, because of the simplicity of our power-law model for the material properties. The experiments clearly show that the observed velocity perturbations are not purely varicose; to extend our theory to include sinuous perturbations would involve introducing even more new parameters so that it would become more difficult to draw robust conclusions about the behaviour of the model. A secondary issue is that our model gives fluid properties (elastic modulus and relaxation time) which depend instantaneously on their flow environment. From physical arguments, and also in order to match the homogenisation theory of [2], a next step might be to extend the model to include a structure factor on which these material properties would depend, and which could itself evolve in reaction to its flow environment.

\section{References}

[1] H. J. Wilson, J. M. Rallison, Instability of channel flow of a shearthinning White-Metzner fluid, Journal of Non-Newtonian Fluid Mechanics 87 (1999) 75-96.

[2] H. Bodiguel, J. Beaumont, A. Machado, L. Martinie, H. Kellay, A. Colin, Flow enhancement due to elastic turbulence in channel flows of shear thinning fluids, Physical Review Letters 114 (2015) 028302. 
[3] C. J. S. Petrie, M. M. Denn, Instabilities in polymer processing, A.I.Ch.E. J. 22 (2) (1976) 209-236.

[4] R. G. Larson, E. S. G. Shaqfeh, S. J. Muller, A purely elastic instability in Taylor-Couette flow, JFM 218 (1990) 573-600.

[5] P. Pakdel, G. H. McKinley, Elastic instability and curved streamlines, PRL 77 (12) (1996) 2459-2462.

[6] E. J. Hinch, O. J. Harris, J. M. Rallison, The instability mechanism for two elastic liquids being coextruded, JNNFM 43 (2-3) (1992) 311-324.

[7] J. C. Miller, J. M. Rallison, Interfacial instability between sheared elastic liquids in a channel, JNNFM 143 (2-3) (2007) 71-87.

[8] O. Radulescu, P. D. Olmsted, Matched asymptotic solutions for the steady banded flow of the diffusive Johnson-Segalman model in various geometries, J. Non-Newt. Fluid Mech. 91 (2000) 143-164.

[9] M. M. Britton, P. T. Callaghan, Two-phase shear band structures at uniform stress, Phys. Rev. Lett. 78 (26) (1997) 4930-4933.

[10] S. M. Fielding, Complex dynamics of shear banded flows, Soft Matter 2 (2007) 1262-1279.

[11] H. J. Wilson, Polymeric fluids (graduate lecture course). Section 4: Microscopic dynamics, http://www.ucl.ac.uk/〜ucahhwi/GM05/lecture45.pdf (2006).

[12] T. C. Ho, M. M. Denn, Stability of plane Poiseuille flow of a highly elastic liquid, Journal of Non-Newtonian Fluid Mechanics 3 (1978) 179.

[13] N. Hoda, Jovanović, S. Kumar, Energy amplification in channel flows of viscoelastic fluids, J. Fluid Mech. 601 (2008) 407-424.

[14] M. R. Jovanović, S. Kumar, Transient growth without inertia, Phys. Fluids 22 (2010) 023101.

[15] B. K. Lieu, M. R. Jovanović, S. Kumar, Worst-case amplification of disturbances in inertialess Couette flow of viscoelastic fluids, J. Fluid Mech. 723 (2013) 232-263. 